\title{
Weighted boundedness of multilinear singular integral operators
}

\author{
Wei-Ping Kuang ${ }^{1}$ and Zhi-Gang Wang ${ }^{2 *}$
}

"Correspondence:

zhigangwang@foxmail.com

${ }^{2}$ School of Mathematics and Statistics, Anyang Normal University,

Anyang, Henan 455000, People's Republic of China

Full list of author information is available at the end of the article

\begin{abstract}
In this paper, we establish the weighted sharp maximal function inequalities for the multilinear singular integral operators. As an application, we obtain the boundedness of the multilinear operators on weighted Lebesgue and Morrey spaces.
\end{abstract}

MSC: 42B20; 42B25

Keywords: multilinear operator; singular integral operator; sharp maximal function; weighted $\mathrm{BMO}$; weighted Lipschitz function

\section{Introduction}

As the development of singular integral operators (see [1-3]), their commutators operators have been well studied. In [4-6], the authors prove that the commutators generated by the singular integral operators and BMO functions are bounded on $L^{p}\left(R^{n}\right)$ for $1<p<\infty$. Chanillo (see [7]) proves a similar result when singular integral operators are replaced by the fractional integral operators. In $[8,9]$, the boundedness for the commutators generated by the singular integral operators and Lipschitz functions on Triebel-Lizorkin and $L^{p}\left(R^{n}\right)(1<p<\infty)$ spaces are obtained. In [10,11], the boundedness for the commutators generated by the singular integral operators and the weighted BMO and Lipschitz functions on $L^{p}\left(R^{n}\right)(1<p<\infty)$ spaces are obtained (also see [12,13]). In [14-17], the authors studied some multilinear singular integral operators as follows (also see [18, 19]):

$$
T^{b}(f)(x)=\int \frac{R_{m+1}(b ; x, y)}{|x-y|^{m}} K(x, y) f(y) d y
$$

and they obtained some variant sharp function estimates and boundedness of the multilinear operators if $D^{\alpha} b \in \mathrm{BMO}\left(R^{n}\right)$ for all $\alpha$ with $|\alpha|=m$. In this paper, we will study the multilinear operator generated by the singular integral operator and the weighted Lipschitz and BMO functions, that is, $D^{\alpha} b \in \mathrm{BMO}(w)$ or $D^{\alpha} b \in \operatorname{Lip}_{\beta}(w)$ for all $\alpha$ with $|\alpha|=m$.

\section{Preliminaries}

First, let us introduce some notations. Throughout this paper, $Q$ will denote a cube of $R^{n}$ with sides parallel to the axes. For any locally integrable function $f$, the sharp maximal function of $f$ is defined by

$$
M^{\#}(f)(x)=\sup _{Q \ni x} \frac{1}{|Q|} \int_{Q}\left|f(y)-f_{Q}\right| d y ;
$$

@2014 Kuang and Wang; licensee Springer. This is an Open Access article distributed under the terms of the Creative Commons Attribution License (http://creativecommons.org/licenses/by/2.0), which permits unrestricted use, distribution, and reproduction in any medium, provided the original work is properly cited. 
here, and in the following, $f_{Q}=|Q|^{-1} \int_{Q} f(x) d x$. It is well known that (see $\left.[1,2]\right)$

$$
M^{\#}(f)(x) \approx \sup _{Q \ni x} \inf _{c \in C} \frac{1}{|Q|} \int_{Q}|f(y)-c| d y
$$

Let

$$
M(f)(x)=\sup _{Q \ni x} \frac{1}{|Q|} \int_{Q}|f(y)| d y .
$$

For $\eta>0$, let $M_{\eta}^{\#}(f)(x)=M^{\#}\left(|f|^{\eta}\right)^{1 / \eta}(x)$ and $M_{\eta}(f)(x)=M\left(|f|^{\eta}\right)^{1 / \eta}(x)$.

For $0<\eta<n, 1 \leq p<\infty$ and the non-negative weight function $w$, set

$$
M_{\eta, p, w}(f)(x)=\sup _{Q \ni x}\left(\frac{1}{w(Q)^{1-p \eta / n}} \int_{Q}|f(y)|^{p} w(y) d y\right)^{1 / p} .
$$

We write $M_{\eta, p, w}(f)=M_{p, w}(f)$ if $\eta=0$.

The $A_{p}$ weight is defined by (see [1]), for $1<p<\infty$,

$$
A_{p}=\left\{w \in L_{\mathrm{loc}}^{1}\left(R^{n}\right): \sup _{Q}\left(\frac{1}{|Q|} \int_{Q} w(x) d x\right)\left(\frac{1}{|Q|} \int_{Q} w(x)^{-1 /(p-1)} d x\right)^{p-1}<\infty\right\}
$$

and

$$
A_{1}=\left\{w \in L_{\mathrm{loc}}^{p}\left(R^{n}\right): M(w)(x) \leq C w(x), \text { a.e. }\right\} .
$$

Given a non-negative weight function $w$. For $1 \leq p<\infty$, the weighted Lebesgue space $L^{p}\left(R^{n}, w\right)$ is the space of functions $f$ such that

$$
\|f\|_{L^{p}(w)}=\left(\int_{R^{n}}|f(x)|^{p} w(x) d x\right)^{1 / p}<\infty .
$$

For $0<\beta<1$ and the non-negative weight function $w$, the weighted Lipschitz space $\operatorname{Lip}_{\beta}(w)$ is the space of functions $b$ such that

$$
\|b\|_{\operatorname{Lip}_{\beta}(w)}=\sup _{Q} \frac{1}{w(Q)^{\beta / n}}\left(\frac{1}{w(Q)} \int_{Q}\left|b(y)-b_{Q}\right|^{p} w(x)^{1-p} d y\right)^{1 / p}<\infty,
$$

and the weighted $\mathrm{BMO}$ space $\mathrm{BMO}(w)$ is the space of functions $b$ such that

$$
\|b\|_{\mathrm{BMO}(w)}=\sup _{Q}\left(\frac{1}{w(Q)} \int_{Q}\left|b(y)-b_{Q}\right|^{p} w(x)^{1-p} d y\right)^{1 / p}<\infty .
$$

\section{Remark}

(1) It is well known that (see $[10,20])$, for $b \in \operatorname{Lip}_{\beta}(w), w \in A_{1}$ and $x \in Q$,

$$
\left|b_{Q}-b_{2^{k} Q}\right| \leq C k\|b\|_{\operatorname{Lip}_{\beta}(w)} w(x) w\left(2^{k} Q\right)^{\beta / n} .
$$


(2) It is well known that (see $[11,20])$, for $b \in \mathrm{BMO}(w), w \in A_{1}$ and $x \in Q$,

$$
\left|b_{Q}-b_{2^{k} Q}\right| \leq C k\|b\|_{\mathrm{BMO}(w)} w(x) .
$$

(3) Let $b \in \operatorname{Lip}_{\beta}(w)$ or $b \in \operatorname{BMO}(w)$ and $w \in A_{1}$. By [20], we know that spaces $\operatorname{Lip}_{\beta}(w)$ or $\mathrm{BMO}(w)$ coincide and the norms $\|b\|_{\operatorname{Lip}_{\beta}(w)}$ or $\|b\|_{\mathrm{BMO}(w)}$ are equivalent with respect to different values $1 \leq p<\infty$.

Definition 1 Let $\varphi$ be a positive, increasing function on $R^{+}$and let there exist a constant $D>0$ such that

$$
\varphi(2 t) \leq D \varphi(t) \quad \text { for } t \geq 0
$$

Let $w$ be a non-negative weight function on $R^{n}$ and $f$ be a locally integrable function on $R^{n}$. Set, for $1 \leq p<\infty$,

$$
\|f\|_{L^{p, \varphi}(w)}=\sup _{x \in R^{n}, d>0}\left(\frac{1}{\varphi(d)} \int_{Q(x, d)}|f(y)|^{p} w(y) d y\right)^{1 / p},
$$

where $Q(x, d)=\left\{y \in R^{n}:|x-y|<d\right\}$. The generalized weighted Morrey space is defined by

$$
L^{p, \varphi}\left(R^{n}, w\right)=\left\{f \in L_{\mathrm{loc}}^{1}\left(R^{n}\right):\|f\|_{L^{p, \varphi}(w)}<\infty\right\} .
$$

If $\varphi(d)=d^{\delta}, \delta>0$, then $L^{p, \varphi}\left(R^{n}, w\right)=L^{p, \delta}\left(R^{n}, w\right)$, which is the classical Morrey spaces (see $[21,22])$. If $\varphi(d)=1$, then $L^{p, \varphi}\left(R^{n}, w\right)=L^{p}\left(R^{n}, w\right)$, which is the weighted Lebesgue spaces (see [1]).

As the Morrey space may be considered as an extension of the Lebesgue space, it is natural and important to study the boundedness of the operator on the Morrey spaces (see [9, 23-28]).

In this paper, we will study the singular integral operators as follows (see [5]).

Definition 2 Let $T: S \rightarrow S^{\prime}$ be a linear operator such that $T$ is bounded on $L^{p}\left(R^{n}\right)$ for $1<p<\infty$ and weak $\left(L^{1}, L^{1}\right)$-bounded and there exists a locally integrable function $K(x, y)$ on $R^{n} \times R^{n} \backslash\left\{(x, y) \in R^{n} \times R^{n}: x=y\right\}$ such that

$$
T(f)(x)=\int_{R^{n}} K(x, y) f(y) d y
$$

for every bounded and compactly supported function $f$, where $K$ satisfies, for fixed $\varepsilon>0$,

$$
|K(x, y)| \leq C|x-y|^{-n}
$$

and

$$
|K(y, x)-K(z, x)|+|K(x, y)-K(x, z)| \leq C|y-z|^{\varepsilon}|x-z|^{-n-\varepsilon}
$$

if $2|y-z| \leq|x-z|$. 
Moreover, let $m$ be the positive integer and $b$ be the function on $R^{n}$. Set

$$
R_{m+1}(b ; x, y)=b(x)-\sum_{|\alpha| \leq m} \frac{1}{\alpha !} D^{\alpha} b(y)(x-y)^{\alpha} .
$$

The multilinear operator related to the operator $T$ is defined by

$$
T^{b}(f)(x)=\int_{R^{n}} \frac{R_{m+1}(b ; x, y)}{|x-y|^{m}} K(x, y) f(y) d y .
$$

Note that the classical Calderón-Zygmund singular integral operator satisfies the conditions of Definition 2 (see $[1,4]$ ) and that the commutator $[b, T](f)=b T(f)-T(b f)$ is a particular operator of the multilinear operator $T^{b}$ if $m=0$. The multilinear operator $T^{b}$ are the non-trivial generalizations of the commutator. It is well known that commutators and multilinear operators are of great interest in harmonic analysis and have been widely studied by many authors (see $[5,6,19])$. The main purpose of this paper is to prove the sharp maximal inequalities for the multilinear operator $T^{b}$. As the application, we obtain the weighted $L^{p}$-norm inequality and Morrey space boundedness for the multilinear operator $T^{b}$.

\section{Theorems and lemmas}

We shall prove the following theorems.

Theorem 1 Let $T$ be the singular integral operator as Definition $2, w \in A_{1}, 0<\eta<1,1<$ $r<\infty$ and $D^{\alpha} b \in \mathrm{BMO}(w)$ for all $\alpha$ with $|\alpha|=m$. Then there exists $a$ constant $C>0$ such that, for any $f \in C_{0}^{\infty}\left(R^{n}\right)$ and $\tilde{x} \in R^{n}$,

$$
M_{\eta}^{\#}\left(T^{b}(f)\right)(\tilde{x}) \leq C \sum_{|\alpha|=m}\left\|D^{\alpha} b\right\|_{\mathrm{BMO}(w)} w(\tilde{x}) M_{r, w}(f)(\tilde{x}) .
$$

Theorem 2 Let $T$ be the singular integral operator as Definition $2, w \in A_{1}, 0<\eta<1,1<$ $r<\infty, 0<\beta<1$ and $D^{\alpha} b \in \operatorname{Lip}_{\beta}(w)$ for all $\alpha$ with $|\alpha|=m$. Then there exists a constant $C>0$ such that, for any $f \in C_{0}^{\infty}\left(R^{n}\right)$ and $\tilde{x} \in R^{n}$,

$$
M_{\eta}^{\#}\left(T^{b}(f)\right)(\tilde{x}) \leq C \sum_{|\alpha|=m}\left\|D^{\alpha} b\right\|_{\operatorname{Lip}_{\beta}(w)} w(\tilde{x}) M_{\beta, r, w}(f)(\tilde{x}) .
$$

Theorem 3 Let $T$ be the singular integral operator as Definition $2, w \in A_{1}, 1<p<\infty$ and $D^{\alpha} b \in \mathrm{BMO}(w)$ for all $\alpha$ with $|\alpha|=m$. Then $T^{b}$ is bounded from $L^{p}\left(R^{n}, w\right)$ to $L^{p}\left(R^{n}, w^{1-p}\right)$.

Theorem 4 Let $T$ be the singular integral operator as Definition 2, $w \in A_{1}, 1<p<\infty$, $0<D<2^{n}$ and $D^{\alpha} b \in \mathrm{BMO}(w)$ for all $\alpha$ with $|\alpha|=m$. Then $T^{b}$ is bounded from $L^{p, \varphi}\left(R^{n}, w\right)$ to $L^{p, \varphi}\left(R^{n}, w^{1-p}\right)$.

Theorem 5 Let $T$ be the singular integral operator as Definition $2, w \in A_{1}, 0<\beta<1,1<$ $p<n / \beta, 1 / q=1 / p-\beta / n$ and $D^{\alpha} b \in \operatorname{Lip}_{\beta}(w)$ for all $\alpha$ with $|\alpha|=m$. Then $T^{b}$ is bounded from $L^{p}\left(R^{n}, w\right)$ to $L^{q}\left(R^{n}, w^{1-q}\right)$. 
Theorem 6 Let $T$ be the singular integral operator as Definition 2, $w \in A_{1}, 0<\beta<1,0<$ $D<2^{n}, 1<p<n / \beta, 1 / q=1 / p-\beta / n$ and $D^{\alpha} b \in \operatorname{Lip}_{\beta}(w)$ for all $\alpha$ with $|\alpha|=m$. Then $T^{b}$ is bounded from $L^{p, \varphi}\left(R^{n}, w\right)$ to $L^{q, \varphi}\left(R^{n}, w^{1-q}\right)$.

To prove the theorems, we need the following lemmas.

Lemma 1 (See [1, p.485]) Let $0<p<q<\infty$ and for any function $f \geq 0$. We define, for $1 / r=1 / p-1 / q$,

$$
\|f\|_{W L^{q}}=\sup _{\lambda>0} \lambda\left|\left\{x \in R^{n}: f(x)>\lambda\right\}\right|^{1 / q}, \quad N_{p, q}(f)=\sup _{Q}\left\|f \chi_{Q}\right\|_{L^{p}} /\left\|\chi_{Q}\right\|_{L^{r}},
$$

where the sup is taken for all measurable sets $Q$ with $0<|Q|<\infty$. Then

$$
\|f\|_{W L^{q}} \leq N_{p, q}(f) \leq(q /(q-p))^{1 / p}\|f\|_{W L^{q}} .
$$

Lemma 2 (See $[1,20])$ Let $0 \leq \eta<n, 1 \leq s<p<n / \eta, 1 / q=1 / p-\eta / n$ and $w \in A_{1}$. Then

$$
\left\|M_{\eta, s, w}(f)\right\|_{L^{q}(w)} \leq C\|f\|_{L^{p}(w)}
$$

Lemma 3 (See [1]) Let $0<p, \eta<\infty$ and $w \in \bigcup_{1 \leq r<\infty} A_{r}$. Then, for any smooth function $f$ for which the left-hand side is finite,

$$
\int_{R^{n}} M_{\eta}(f)(x)^{p} w(x) d x \leq C \int_{R^{n}} M_{\eta}^{\#}(f)(x)^{p} w(x) d x .
$$

Lemma 4 (See [1]) Let $0<p<\infty, 0<\eta<\infty, 0<D<2^{n}$ and $w \in A_{1}$. Then, for any smooth function $f$ for which the left-hand side is finite,

$$
\left\|M_{\eta}(f)\right\|_{L^{p, \varphi}(w)} \leq C\left\|M_{\eta}^{\#}(f)\right\|_{L^{p, \varphi}(w)} .
$$

Lemma 5 (See [20]) Let $0 \leq \eta<n, 0<D<2^{n}, 1 \leq s<p<n / \eta, 1 / q=1 / p-\eta / n$ and $w \in A_{1}$. Then

$$
\left\|M_{\eta, s, w}(f)\right\|_{L^{q, \varphi}(w)} \leq C\|f\|_{L^{p, \varphi}(w)} .
$$

Lemma 6 (See [16]) Let b be a function on $R^{n}$ and $D^{\alpha} A \in L^{q}\left(R^{n}\right)$ for all $\alpha$ with $|\alpha|=m$ and any $q>n$. Then

$$
\left|R_{m}(b ; x, y)\right| \leq C|x-y|^{m} \sum_{|\alpha|=m}\left(\frac{1}{|\tilde{Q}(x, y)|} \int_{\tilde{Q}(x, y)}\left|D^{\alpha} b(z)\right|^{q} d z\right)^{1 / q},
$$

where $\tilde{Q}$ is the cube centered at $x$ and having side length $5 \sqrt{n}|x-y|$.

\section{Proofs of theorems}

Proof of Theorem 1 It suffices to prove for $f \in C_{0}^{\infty}\left(R^{n}\right)$ and some constant $C_{0}$, the following inequality holds:

$$
\left(\frac{1}{|Q|} \int_{Q}\left|T^{b}(f)(x)-C_{0}\right|^{\eta} d x\right)^{1 / \eta} \leq C \sum_{|\alpha|=m}\left\|D^{\alpha} b\right\|_{\mathrm{BMO}(w)} w(\tilde{x}) M_{s, w}(f)(\tilde{x}) .
$$


Fix a cube $Q=Q\left(x_{0}, d\right)$ and $\tilde{x} \in Q$. Let $\tilde{Q}=5 \sqrt{n} Q$ and $\tilde{b}(x)=b(x)-\sum_{|\alpha|=m} \frac{1}{\alpha !}\left(D^{\alpha} b\right)_{\tilde{Q}} x^{\alpha}$, then $R_{m}(b ; x, y)=R_{m}(\tilde{b} ; x, y)$ and $D^{\alpha} \tilde{b}=D^{\alpha} b-\left(D^{\alpha} b\right)_{\tilde{Q}}$ for $|\alpha|=m$. We write, for $f_{1}=f \chi_{\tilde{Q}}$ and $f_{2}=f \chi_{R^{n} \backslash \tilde{Q}}$,

$$
\begin{aligned}
T^{b}(f)(x)= & \int_{R^{n}} \frac{R_{m}(\tilde{b} ; x, y)}{|x-y|^{m}} K(x, y) f_{1}(y) d y-\sum_{|\alpha|=m} \frac{1}{\alpha !} \int_{R^{n}} \frac{(x-y)^{\alpha} D^{\alpha} \tilde{b}(y)}{|x-y|^{m}} K(x, y) f_{1}(y) d y \\
& +\int_{R^{n}} \frac{R_{m+1}(\tilde{b} ; x, y)}{|x-y|^{m}} K(x, y) f_{2}(y) d y \\
= & T\left(\frac{R_{m}(\tilde{b} ; x, \cdot)}{|x-\cdot|^{m}} f_{1}\right)-T\left(\sum_{|\alpha|=m} \frac{1}{\alpha !} \frac{(x-\cdot)^{\alpha} D^{\alpha} \tilde{b}}{|x-\cdot|^{m}} f_{1}\right)+T^{\tilde{b}}\left(f_{2}\right)(x),
\end{aligned}
$$

then

$$
\begin{aligned}
& \left(\frac{1}{|Q|} \int_{Q}\left|T^{b}(f)(x)-T^{b}\left(f_{2}\right)\left(x_{0}\right)\right|^{\eta} d x\right)^{1 / \eta} \\
& \leq C\left(\frac{1}{|Q|} \int_{Q}\left|T\left(\frac{R_{m}(\tilde{b} ; x, \cdot)}{|x-\cdot|^{m}} f_{1}\right)\right|^{\eta} d x\right)^{1 / \eta} \\
& \quad+C\left(\frac{1}{|Q|} \int_{Q}\left|T\left(\sum_{|\alpha|=m} \frac{(x-\cdot)^{\alpha} D^{\alpha} \tilde{b}}{|x-\cdot|^{m}} f_{1}\right)\right|^{\eta} d x\right)^{1 / \eta} \\
& \quad+C\left(\frac{1}{|Q|} \int_{Q}\left|T^{\tilde{b}}\left(f_{2}\right)(x)-T^{\tilde{b}}\left(f_{2}\right)\left(x_{0}\right)\right|^{\eta} d x\right)^{1 / \eta} \\
& =I_{1}+I_{2}+I_{3} .
\end{aligned}
$$

For $I_{1}$, noting that $w \in A_{1}, w$ satisfies the reverse of Hölder's inequality:

$$
\left(\frac{1}{|Q|} \int_{Q} w(x)^{p_{0}} d x\right)^{1 / p_{0}} \leq \frac{C}{|Q|} \int_{Q} w(x) d x
$$

for all cube $Q$ and some $1<p_{0}<\infty$ (see [1]). We take $q=r p_{0} /\left(r+p_{0}-1\right)$ in Lemma 6 and have $1<q<r$ and $p_{0}=q(r-1) /(r-q)$, then by the Lemma 6 and Hölder's inequality, we obtain

$$
\begin{aligned}
& \left|R_{m}(\tilde{b} ; x, y)\right| \\
& \leq C|x-y|^{m} \sum_{|\alpha|=m}\left(\frac{1}{|\tilde{Q}(x, y)|} \int_{\tilde{Q}(x, y)}\left|D^{\alpha} \tilde{b}(z)\right|^{q} d z\right)^{1 / q} \\
& \leq C|x-y|^{m} \sum_{|\alpha|=m}|\tilde{Q}|^{-1 / q}\left(\int_{\tilde{Q}(x, y)}\left|D^{\alpha} \tilde{b}(z)\right|^{q} w(z)^{q(1-r) / r} w(z)^{q(r-1) / r} d z\right)^{1 / q} \\
& \leq C|x-y|^{m} \sum_{|\alpha|=m}|\tilde{Q}|^{-1 / q}\left(\int_{\tilde{Q}(x, y)}\left|D^{\alpha} \tilde{b}(z)\right|^{r} w(z)^{1-r} d z\right)^{1 / r} \\
& \quad \times\left(\int_{\tilde{Q}(x, y)} w(z)^{q(r-1) /(r-q)} d z\right)^{(r-q) /(r q)}
\end{aligned}
$$




$$
\begin{aligned}
\leq & C|x-y|^{m} \sum_{|\alpha|=m}|\tilde{Q}|^{-1 / q}\left\|D^{\alpha} b\right\|_{\mathrm{BMO}(w)} w(\tilde{Q})^{1 / r}|\tilde{Q}|^{(r-q) / r q} \\
& \times\left(\frac{1}{|\tilde{Q}(x, y)|} \int_{\tilde{Q}(x, y)} w(z)^{p 0} d z\right)^{(r-q) / r q} \\
\leq & C|x-y|^{m} \sum_{|\alpha|=m}\left\|D^{\alpha} b\right\|_{\mathrm{BMO}(w)}|\tilde{Q}|^{-1 / q} w(\tilde{Q})^{1 / r}|\tilde{Q}|^{1 / q-1 / r}\left(\frac{1}{|\tilde{Q}(x, y)|} \int_{\tilde{Q}(x, y)} w(z) d z\right)^{(r-1) / r} \\
\leq & C|x-y|^{m} \sum_{|\alpha|=m}\left\|D^{\alpha} b\right\|_{\mathrm{BMO}(w)}|\tilde{Q}|^{-1 / q} w(\tilde{Q})^{1 / r}|\tilde{Q}|^{1 / q-1 / r} w(\tilde{Q})^{1-1 / r}|\tilde{Q}|^{1 / r-1} \\
\leq & C|x-y|^{m} \sum_{|\alpha|=m}\left\|D^{\alpha} b\right\|_{\mathrm{BMO}(w)} \frac{w(\tilde{Q})}{|\tilde{Q}|} \\
\leq & C|x-y|^{m} \sum_{|\alpha|=m}\left\|D^{\alpha} b\right\|_{\mathrm{BMO}(w)} w(\tilde{x})
\end{aligned}
$$

thus, by the $L^{s}$-boundedness of $T$ (see Lemma 2) for $1<s<r$ and $w \in A_{1} \subseteq A_{r / s}$, we obtain

$$
\begin{aligned}
I_{1} \leq & \frac{C}{|Q|} \int_{Q}\left|T\left(\frac{R_{m}(\tilde{b} ; x, \cdot)}{|x-\cdot|^{m}} f_{1}\right)\right| d x \\
\leq & C \sum_{|\alpha|=m}\left\|D^{\alpha} b\right\|_{\mathrm{BMO}(w)} w(\tilde{x})\left(\frac{1}{|Q|} \int_{R^{n}}\left|T\left(f_{1}\right)(x)\right|^{s} d x\right)^{1 / s} \\
\leq & C \sum_{|\alpha|=m}\left\|D^{\alpha} b\right\|_{\mathrm{BMO}(w)} w(\tilde{x})|Q|^{-1 / s}\left(\int_{R^{n}}\left|f_{1}(x)\right|^{s} d x\right)^{1 / s} \\
\leq & C \sum_{|\alpha|=m}\left\|D^{\alpha} b\right\|_{\mathrm{BMO}(w)} w(\tilde{x})|Q|^{-1 / s}\left(\int_{\tilde{Q}}|f(x)|^{s} w(x)^{s / r} w(x)^{-s / r} d x\right)^{1 / s} \\
\leq & C \sum_{|\alpha|=m}\left\|D^{\alpha} b\right\|_{\mathrm{BMO}(w)} w(\tilde{x})|Q|^{-1 / s}\left(\int_{\tilde{Q}}|f(x)|^{r} w(x) d x\right)^{1 / r}\left(\int_{\tilde{Q}} w(x)^{-s /(r-s)} d x\right)^{(r-s) / r s} \\
\leq & C \sum_{|\alpha|=m}\left\|D^{\alpha} b\right\|_{\mathrm{BMO}(w)} w(\tilde{x})|Q|^{-1 / s} w(\tilde{Q})^{1 / r}\left(\frac{1}{w(\tilde{Q})} \int_{\tilde{Q}}|f(x)|^{r} w(x) d x\right)^{1 / r} \\
& \times\left(\frac{1}{|\tilde{Q}|} \int_{\tilde{Q}} w(x)^{-s /(r-s)} d x\right)^{(r-s) / r s}\left(\frac{1}{|\tilde{Q}|} \int_{\tilde{Q}} w(x) d x\right)^{1 / r}|\tilde{Q}|^{1 / s} w(\tilde{Q})^{-1 / r} \\
\leq & C \sum_{|\alpha|=m}\left\|D^{\alpha} b\right\|_{\mathrm{BMO}(w)} w(\tilde{x}) M_{r, w}(f)(\tilde{x}) .
\end{aligned}
$$

For $I_{2}$, by the weak $\left(L^{1}, L^{1}\right)$ boundedness of $T$ (see Lemma 2) and Kolmogoro's inequality (see Lemma 1), we obtain

$$
\begin{aligned}
I_{2} & \leq C \sum_{|\alpha|=m}\left(\frac{1}{|Q|} \int_{Q}\left|T\left(D^{\alpha} \tilde{b} f_{1}\right)(x)\right|^{\eta} d x\right)^{1 / \eta} \\
& \leq C \sum_{|\alpha|=m} \frac{|Q|^{1 / \eta-1}}{|Q|^{1 / \eta}} \frac{\left\|T\left(D^{\alpha} \tilde{b} f_{1}\right) \chi_{Q}\right\|_{L^{\eta}}}{\left\|\chi_{Q}\right\|_{L^{\eta /(1-\eta)}}} \\
& \leq C \sum_{|\alpha|=m} \frac{1}{|Q|}\left\|T\left(D^{\alpha} \tilde{b} f_{1}\right)\right\|_{W L^{1}}
\end{aligned}
$$




$$
\begin{aligned}
& \leq C \sum_{|\alpha|=m} \frac{1}{|Q|} \int_{R^{n}}\left|D^{\alpha} \tilde{b}(x) f_{1}(x)\right| d x \\
& \leq C \sum_{|\alpha|=m} \frac{1}{|Q|} \int_{\tilde{Q}}\left|D^{\alpha} b(x)-\left(D^{\alpha} b\right)_{\tilde{Q}}\right| w(x)^{-1 / r}|f(x)| w(x)^{1 / r} d x \\
& \leq C \sum_{|\alpha|=m} \frac{1}{|Q|}\left(\int_{\tilde{Q}}\left|\left(D^{\alpha} b(x)-\left(D^{\alpha} b\right)_{\tilde{Q}}\right)\right|^{r^{\prime}} w(x)^{1-r^{\prime}} d x\right)^{1 / r^{\prime}}\left(\int_{\tilde{Q}}|f(x)|^{r} w(x) d x\right)^{1 / r} \\
& \leq C \sum_{|\alpha|=m} \frac{1}{|Q|}\left\|D^{\alpha} b\right\|_{\mathrm{BMO}(w)} w(\tilde{Q})^{1 / r^{\prime}} w(\tilde{Q})^{1 / r}\left(\frac{1}{w(\tilde{Q})} \int_{\tilde{Q}}|f(x)|^{r} w(x) d x\right)^{1 / r} \\
& \leq C \sum_{|\alpha|=m}\left\|D^{\alpha} b\right\|_{\mathrm{BMO}(w)} \frac{w(\tilde{Q})}{|\tilde{Q}|} M_{r, w}(f)(\tilde{x}) \\
& \leq C \sum_{|\alpha|=m}\left\|D^{\alpha} b\right\|_{\mathrm{BMO}(w)} w(\tilde{x}) M_{r, w}(f)(\tilde{x}) .
\end{aligned}
$$

For $I_{3}$, note that $|x-y| \approx\left|x_{0}-y\right|$ for $x \in Q$ and $y \in R^{n} \backslash Q$, we write

$$
\begin{aligned}
& T^{\tilde{b}}\left(f_{2}\right)(x)-T^{\tilde{b}}\left(f_{2}\right)\left(x_{0}\right) \\
& =\int_{R^{n}}\left(R_{m}(\tilde{b} ; x, y)-R_{m}\left(\tilde{b} ; x_{0}, y\right)\right) \frac{K(x, y)}{|x-y|^{m}}\left|f_{2}(y)\right| d y \\
& \quad+\int_{R^{n}}\left(\frac{K(x, y)}{|x-y|^{m}}-\frac{K\left(x_{0}, y\right)}{\left|x_{0}-y\right|^{m}}\right) R_{m}\left(\tilde{b} ; x_{0}, y\right) f_{2}(y) d y \\
& \quad+\sum_{|\alpha|=m} \frac{1}{\alpha !} \int_{R^{n}}\left(\frac{K(x, y)}{|x-y|^{m}}-\frac{K\left(x_{0}, y\right)}{\left|x_{0}-y\right|^{m}}\right)(x-y)^{\alpha} D^{\alpha} \tilde{b}(y) f_{2}(y) d y \\
& \quad+\sum_{|\alpha|=m} \frac{1}{\alpha !} \int_{R^{n}}\left(\frac{(x-y)^{\alpha}}{|x-y|^{m}}-\frac{\left(x_{0}-y\right)^{\alpha}}{\left|x_{0}-y\right|^{m}}\right) K\left(x_{0}, y\right) D^{\alpha} \tilde{b}(y) f_{2}(y) d y \\
& =I_{3}^{(1)}(x)+I_{3}^{(2)}(x)+I_{3}^{(3)}(x)+I_{3}^{(4)}(x) .
\end{aligned}
$$

For $I_{3}^{(1)}(x)$, by the formula (see [16]):

$$
R_{m}(\tilde{b} ; x, y)-R_{m}\left(\tilde{b} ; x_{0}, y\right)=\sum_{|\gamma|<m} \frac{1}{\gamma !} R_{m-|\gamma|}\left(D^{\gamma} \tilde{b} ; x, x_{0}\right)(x-y)^{\gamma}
$$

and Lemma 6, we have, similar to the proof of $I_{1}$,

$$
\left|R_{m}(\tilde{b} ; x, y)-R_{m}\left(\tilde{b} ; x_{0}, y\right)\right| \leq C \sum_{|\gamma|<m} \sum_{|\alpha|=m}\left|x-x_{0}\right|^{m-|\gamma|}|x-y|^{|\gamma|}\left\|D^{\alpha} b\right\|_{\mathrm{BMO}(w)} w(\tilde{x}),
$$

thus, by $w \in A_{1} \subseteq A_{r}$,

$$
\begin{aligned}
& \left|I_{3}^{(1)}(x)\right| \\
& \quad \leq \sum_{k=0}^{\infty} \int_{2^{k+1} \tilde{Q} \backslash 2^{k} \tilde{Q}}\left|R_{m}(\tilde{b} ; x, y)-R_{m}\left(\tilde{b} ; x_{0}, y\right)\right| \frac{|K(x, y)|}{|x-y|^{m}}|f(y)| d y \\
& \quad \leq C \sum_{|\alpha|=m}\left\|D^{\alpha} b\right\|_{\mathrm{BMO}(w)} w(\tilde{x}) \sum_{k=0}^{\infty} \int_{2^{k+1} \tilde{Q} \backslash 2^{k} \tilde{Q}} \frac{\left|x-x_{0}\right|}{\left|x_{0}-y\right|^{n+1}}|f(y)| d y
\end{aligned}
$$


Kuang and Wang Journal of Inequalities and Applications 2014, 2014:115

http://www.journalofinequalitiesandapplications.com/content/2014/1/115

Page 9 of 15

$$
\begin{aligned}
\leq & C \sum_{|\alpha|=m}\left\|D^{\alpha} b\right\|_{\mathrm{BMO}(w)} w(\tilde{x}) \sum_{k=1}^{\infty} \frac{d}{\left(2^{k} d\right)^{n+1}} \int_{2^{k} \tilde{Q}}|f(y)| w(y)^{1 / r} w(y)^{-1 / r} d y \\
\leq & C \sum_{|\alpha|=m}\left\|D^{\alpha} b\right\|_{\mathrm{BMO}(w)} w(\tilde{x}) \\
& \times \sum_{k=1}^{\infty} \frac{d}{\left(2^{k} d\right)^{n+1}}\left(\int_{2^{k} \tilde{Q}}|f(y)|^{r} w(y) d y\right)^{1 / r}\left(\int_{2^{k} \tilde{Q}} w(y)^{-1 /(r-1)} d y\right)^{(r-1) / r} \\
\leq & C \sum_{|\alpha|=m}\left\|D^{\alpha} b\right\|_{\mathrm{BMO}(w)} w(\tilde{x}) \sum_{k=1}^{\infty} \frac{d}{\left(2^{k} d\right)^{n+1}} w\left(2^{k} \tilde{Q}\right)^{1 / r}\left(\frac{1}{w\left(2^{k} \tilde{Q}\right)} \int_{2^{k} \tilde{Q}}|f(y)|^{r} w(y) d x\right)^{1 / r} \\
& \left.\times \sum_{\left|2^{k} \tilde{Q}\right|} \int_{2^{k} \tilde{Q}} w(y)^{-1 /(r-1)} d y\right)^{(r-1) / r}\left(\frac{1}{\left|2^{k} \tilde{Q}\right|} \int_{2^{k} \tilde{Q}} w(y) d y\right)^{1 / r}\left|2^{k} \tilde{Q}\right| w\left(2^{k} \tilde{Q}\right)^{-1 / r} \\
\leq & C \sum_{|\alpha|=m}\left\|D^{\alpha} b\right\|_{\mathrm{BMO}(w)} w(\tilde{x}) M_{r, w}(f)(\tilde{x}) \sum_{k=1}^{\infty} 2^{-k} \\
\leq & C \sum_{|\alpha|=m}\left\|D^{\alpha} b\right\|_{\mathrm{BMO}(w)} w(\tilde{x}) M_{r, w}(f)(\tilde{x}) .
\end{aligned}
$$

For $I_{3}^{(2)}(x)$, by the conditions of $K$, we get

$$
\begin{aligned}
\left|I_{3}^{(2)}(x)\right| & \leq C \sum_{k=0}^{\infty} \int_{2^{k+1} \tilde{Q} \backslash 2^{k} \tilde{Q} \mid}\left|\frac{K(x, y)}{|x-y|^{m}}-\frac{K\left(x_{0}, y\right)}{\left|x_{0}-y\right|^{m}}\right|\left|R_{m}\left(\tilde{b} ; x_{0}, y\right)\right| f(y) \mid d y \\
& \leq C \sum_{|\alpha|=m}\left\|D^{\alpha} b\right\|_{\mathrm{BMO}(w)} w(\tilde{x}) \sum_{k=0}^{\infty} \int_{2^{k+1} \tilde{Q} \backslash 2^{k} \tilde{Q}}\left(\frac{\left|x-x_{0}\right|}{\left|x_{0}-y\right|^{n+1}}+\frac{\left|x-x_{0}\right|^{\varepsilon}}{\left|x_{0}-y\right|^{n+\varepsilon}}\right)|f(y)| d y \\
& \leq C \sum_{|\alpha|=m}\left\|D^{\alpha} b\right\|_{\mathrm{BMO}(w)} w(\tilde{x}) \sum_{k=1}^{\infty}\left(\frac{d}{\left(2^{k} d\right)^{n+1}}+\frac{d^{\varepsilon}}{\left(2^{k} d\right)^{n+\varepsilon}}\right) \int_{2^{k} \tilde{Q}}|f(y)| d y \\
& \leq C \sum_{|\alpha|=m}\left\|D^{\alpha} b\right\|_{\mathrm{BMO}(w)} w(\tilde{x}) M_{r, w}(f)(\tilde{x}) \sum_{k=1}^{\infty}\left(2^{-k}+2^{-k \varepsilon}\right) \\
& \leq C \sum_{|\alpha|=m}\left\|D^{\alpha} b\right\|_{\mathrm{BMO}(w)} w(\tilde{x}) M_{r, w}(f)(\tilde{x}) .
\end{aligned}
$$

Similarly, we have

$$
\begin{aligned}
& \left|I_{3}^{(3)}(x)\right|+\left|I_{3}^{(4)}(x)\right| \\
& \leq C \sum_{|\alpha|=m} \sum_{k=0}^{\infty} \int_{2^{k+1} \tilde{Q} \backslash 2^{k} \tilde{Q}}\left(\frac{d}{\left(2^{k} d\right)^{n+1}}+\frac{d^{\varepsilon}}{\left(2^{k} d\right)^{n+\varepsilon}}\right)|f(y)|\left|D^{\alpha} \tilde{b}(y)\right| d y \\
& \leq C \\
& \quad \sum_{|\alpha|=m} \sum_{k=1}^{\infty}\left(\frac{d}{\left(2^{k} d\right)^{n+1}}+\frac{d^{\varepsilon}}{\left(2^{k} d\right)^{n+\varepsilon}}\right) \\
& \quad \times \int_{2^{k} \tilde{Q}}\left|D^{\alpha} b(y)-\left(D^{\alpha} b\right)_{2^{k} \tilde{Q}}\right| w(y)^{-1 / r}|f(y)| w(y)^{1 / r} d y \\
& \quad+C \sum_{|\alpha|=m} \sum_{k=1}^{\infty}\left(\frac{d}{\left(2^{k} d\right)^{n+1}}+\frac{d^{\varepsilon}}{\left(2^{k} d\right)^{n+\varepsilon}}\right)
\end{aligned}
$$


Kuang and Wang Journal of Inequalities and Applications 2014, 2014:115

Page 10 of 15

http://www.journalofinequalitiesandapplications.com/content/2014/1/115

$$
\begin{aligned}
& \times \int_{2^{k} \tilde{Q}}\left|\left(D^{\alpha} b\right)_{2^{k} \tilde{Q}}-\left(D^{\alpha} b\right)_{\tilde{Q}}\right||f(y)| w(y)^{1 / r} w(y)^{-1 / r} d y \\
& \leq C \sum_{|\alpha|=m} \sum_{k=1}^{\infty}\left(\frac{d}{\left(2^{k} d\right)^{n+1}}+\frac{d^{\varepsilon}}{\left(2^{k} d\right)^{n+\varepsilon}}\right)\left(\int_{2^{k} \tilde{Q}}\left|\left(D^{\alpha} b(y)-\left(D^{\alpha} b\right)_{2^{k} \tilde{Q}}\right)\right|^{r^{\prime}} w(y)^{1-r^{\prime}} d y\right)^{1 / r^{\prime}} \\
& \times\left(\int_{2^{k} \tilde{Q}}|f(y)|^{r} w(y) d y\right)^{1 / r} \\
& +C \sum_{|\alpha|=m}\left\|D^{\alpha} b\right\|_{\mathrm{BMO}(w)} w(\tilde{x}) \sum_{k=1}^{\infty} k\left(\frac{d}{\left(2^{k} d\right)^{n+1}}+\frac{d^{\varepsilon}}{\left(2^{k} d\right)^{n+\varepsilon}}\right)\left(\int_{2^{k} \tilde{Q}}|f(y)|^{r} w(y) d x\right)^{1 / r} \\
& \times\left(\frac{1}{\left|2^{k} \tilde{Q}\right|} \int_{2^{k} \tilde{Q}} w(y)^{-1 /(r-1)} d y\right)^{(r-1) / r}\left(\frac{1}{\left|2^{k} \tilde{Q}\right|} \int_{2^{k} \tilde{Q}} w(y) d y\right)^{1 / r}\left|2^{k} \tilde{Q}\right| w\left(2^{k} \tilde{Q}\right)^{-1 / r} \\
& \leq C \sum_{|\alpha|=m}\left\|D^{\alpha} b\right\|_{\mathrm{BMO}(w)} \sum_{k=1}^{\infty}\left(2^{-k}+2^{-k \varepsilon}\right) \frac{w\left(2^{k} \tilde{Q}\right)}{\left|2^{k} \tilde{Q}\right|}\left(\frac{1}{w\left(2^{k} \tilde{Q}\right)} \int_{2^{k} \tilde{Q}}|f(y)|^{r} w(y) d x\right)^{1 / r} \\
& +C \sum_{|\alpha|=m}\left\|D^{\alpha} b\right\|_{\mathrm{BMO}(w)} w(\tilde{x}) \sum_{k=1}^{\infty} k\left(2^{-k}+2^{-k \varepsilon}\right)\left(\frac{1}{w\left(2^{k} \tilde{Q}\right)} \int_{2^{k} \tilde{Q}}|f(y)|^{r} w(y) d x\right)^{1 / r} \\
& \leq C \sum_{|\alpha|=m}\left\|D^{\alpha} b\right\|_{\mathrm{BMO}(w)} w(\tilde{x}) M_{r, w}(f)(\tilde{x}) .
\end{aligned}
$$

Thus

$$
I_{3} \leq C \sum_{|\alpha|=m}\left\|D^{\alpha} b\right\|_{\mathrm{BMO}(w)} w(\tilde{x}) M_{r, w}(f)(\tilde{x})
$$

These complete the proof of Theorem 1.

Proof of Theorem 2 It suffices to prove for $f \in C_{0}^{\infty}\left(R^{n}\right)$ and some constant $C_{0}$, the following inequality holds:

$$
\left(\frac{1}{|Q|} \int_{Q}\left|T^{b}(f)(x)-C_{0}\right|^{\eta} d x\right)^{1 / \eta} \leq C \sum_{|\alpha|=m}\left\|D^{\alpha} b\right\|_{\operatorname{Lip}_{\beta}(w)} w(\tilde{x}) M_{\beta, r, w}(f)(\tilde{x}) .
$$

Fix a cube $Q=Q\left(x_{0}, d\right)$ and $\tilde{x} \in Q$. Similar to the proof of Theorem 1 , we have, for $f_{1}=f \chi_{\tilde{Q}}$ and $f_{2}=f \chi_{R^{n} \backslash \tilde{Q}}$,

$$
\begin{aligned}
& \left(\frac{1}{|Q|} \int_{Q}\left|T^{b}(f)(x)-T^{\tilde{b}}\left(f_{2}\right)\left(x_{0}\right)\right|^{\eta} d x\right)^{1 / \eta} \\
& \leq C\left(\frac{1}{|Q|} \int_{Q}\left|T\left(\frac{R_{m}(\tilde{b} ; x, \cdot)}{|x-\cdot|^{m}} f_{1}\right)\right|^{\eta} d x\right)^{1 / \eta} \\
& \quad+C\left(\frac{1}{|Q|} \int_{Q}\left|T\left(\sum_{|\alpha|=m} \frac{(x-\cdot)^{\alpha} D^{\alpha} \tilde{b}}{|x-\cdot|^{m}} f_{1}\right)\right|^{\eta} d x\right)^{1 / \eta} \\
& \quad+C\left(\frac{1}{|Q|} \int_{Q}\left|T^{\tilde{b}}\left(f_{2}\right)(x)-T^{\tilde{b}}\left(f_{2}\right)\left(x_{0}\right)\right|^{\eta} d x\right)^{1 / \eta} \\
& =J_{1}+J_{2}+J_{3} .
\end{aligned}
$$


For $J_{1}$ and $J_{2}$, by using the same argument as in the proof of Theorem 1, we get

$$
\begin{aligned}
\left|R_{m}(\tilde{b} ; x, y)\right| & \\
\leq & C|x-y|^{m} \sum_{|\alpha|=m}|\tilde{Q}|^{-1 / q}\left(\int_{\tilde{Q}(x, y)}\left|D^{\alpha} \tilde{b}(z)\right|^{q} w(z)^{q(1-r) / r} w(z)^{q(r-1) / r} d z\right)^{1 / q} \\
\leq & C|x-y|^{m} \\
& \times \sum_{|\alpha|=m}|\tilde{Q}|^{-1 / q}\left(\int_{\tilde{Q}(x, y)}\left|D^{\alpha} \tilde{b}(z)\right|^{r} w(z)^{1-r} d z\right)^{1 / r}\left(\int_{\tilde{Q}(x, y)} w(z)^{q(r-1) /(r-q)} d z\right)^{(r-q) / r q} \\
\leq & C|x-y|^{m} \\
& \times \sum_{|\alpha|=m}|\tilde{Q}|^{-1 / q}\left\|D^{\alpha} b\right\|_{\operatorname{Lip}_{\beta}(w)} w(\tilde{Q})^{\beta / n+1 / r}|\tilde{Q}|^{(r-q) / r q}\left(\frac{1}{|\tilde{Q}(x, y)|} \int_{\tilde{Q}(x, y)} w(z)^{p_{0}} d z\right)^{(r-q) / r q} \\
\leq & C|x-y|^{m} \\
& \times \sum_{|\alpha|=m}\left\|D^{\alpha} b\right\|_{\operatorname{Lip}_{\beta}(w)}|\tilde{Q}|^{-1 / q} w(\tilde{Q})^{\beta / n+1 / r}|\tilde{Q}|^{1 / q-1 / r}\left(\frac{1}{|\tilde{Q}(x, y)|} \int_{\tilde{Q}(x, y)} w(z) d z\right)^{(r-1) / r} \\
\leq & C|x-y|^{m} \sum_{|\alpha|=m}\left\|D^{\alpha} b\right\|_{\operatorname{Lip}_{\beta}(w)}|\tilde{Q}|^{-1 / q} w(\tilde{Q})^{\beta / n+1 / r}|\tilde{Q}|^{1 / q-1 / r} w(\tilde{Q})^{1-1 / r}|\tilde{Q}|^{1 / r-1} \\
\leq & C|x-y|^{m} \sum_{|\alpha|=m}\left\|D^{\alpha} b\right\|_{\operatorname{Lip}_{\beta}(w)} \frac{w(\tilde{Q})^{\beta / n+1}}{|\tilde{Q}|} \\
\leq & C|x-y|^{m} \sum_{|\alpha|=m}\left\|D^{\alpha} b\right\|_{\operatorname{Lip}_{\beta}(w)} w(\tilde{Q})^{\beta / n} w(\tilde{x}),
\end{aligned}
$$

thus

$$
\begin{aligned}
J_{1} \leq & C \sum_{|\alpha|=m}\left\|D^{\alpha} b\right\|_{\operatorname{Lip}_{\beta}(w)} w(\tilde{Q})^{\beta / n} w(\tilde{x})|Q|^{-1 / s}\left(\int_{R^{n}}\left|f_{1}(x)\right|^{s} d x\right)^{1 / s} \\
\leq & C \sum_{|\alpha|=m}\left\|D^{\alpha} b\right\|_{\operatorname{Lip}_{\beta}(w)} w(\tilde{Q})^{\beta / n} w(\tilde{x})|Q|^{-1 / s}\left(\int_{\tilde{Q}}|f(x)|^{r} w(x) d x\right)^{1 / r} \\
& \times\left(\int_{\tilde{Q}} w(x)^{-s /(r-s)} d x\right)^{(r-s) / r s} \\
\leq & C \sum_{|\alpha|=m}\left\|D^{\alpha} b\right\|_{\operatorname{Lip}_{\beta}(w)} w(\tilde{x})|\tilde{Q}|^{-1 / s} w(\tilde{Q})^{1 / r}\left(\frac{1}{w(\tilde{Q})^{1-r \beta / n}} \int_{\tilde{Q}}|f(x)|^{r} w(x) d x\right)^{1 / r} \\
& \times\left(\frac{1}{|\tilde{Q}|} \int_{\tilde{Q}} w(x)^{-s /(r-s)} d x\right)^{(r-s) / r s}\left(\frac{1}{|\tilde{Q}|} \int_{\tilde{Q}} w(x) d x\right)^{1 / r}|\tilde{Q}|^{1 / s} w(\tilde{Q})^{-1 / r} \\
\leq & C \sum_{|\alpha|=m}\left\|D^{\alpha} b\right\|_{\operatorname{Lip}_{\beta}(w)} w(\tilde{x}) M_{\beta, r, w}(f)(\tilde{x}), \\
J_{2} \leq & C \sum_{|\alpha|=m} \frac{1}{|Q|} \int_{\tilde{Q}}\left|D^{\alpha} b(x)-\left(D^{\alpha} b\right)_{\tilde{Q}}\right| w(x)^{-1 / r}|f(x)| w(x)^{1 / r} d x \\
\leq & C \sum_{|\alpha|=m} \frac{1}{|Q|}\left(\int_{\tilde{Q}}\left|\left(D^{\alpha} b(x)-\left(D^{\alpha} b\right)_{\tilde{Q}}\right)\right|^{r^{\prime}} w(x)^{1-r^{\prime}} d x\right)^{1 / r^{\prime}}\left(\int_{\tilde{Q}}|f(x)|^{r} w(x) d x\right)^{1 / r}
\end{aligned}
$$


Kuang and Wang Journal of Inequalities and Applications 2014, 2014:115

http://www.journalofinequalitiesandapplications.com/content/2014/1/115

Page 12 of 15

$$
\begin{aligned}
& \leq C \sum_{|\alpha|=m} \frac{1}{|Q|}\left\|D^{\alpha} b\right\|_{\operatorname{Lip}_{\beta}(w)} w(\tilde{Q})^{\beta / n+1 / r^{\prime}} w(\tilde{Q})^{1 / r-\beta / n}\left(\frac{1}{w(\tilde{Q})^{1-r \beta / n}} \int_{\tilde{Q}}|f(x)|^{r} w(x) d x\right)^{1 / r} \\
& \leq C \sum_{|\alpha|=m}\left\|D^{\alpha} b\right\|_{\operatorname{Lip}_{\beta}(w)} \frac{w(\tilde{Q})}{|\tilde{Q}|} M_{\beta, r, w}(f)(\tilde{x}) \\
& \leq C \sum_{|\alpha|=m}\left\|D^{\alpha} b\right\|_{\operatorname{Lip}_{\beta}(w)} w(\tilde{x}) M_{\beta, r, w}(f)(\tilde{x}) .
\end{aligned}
$$

For $J_{3}$, we have

$$
\begin{aligned}
& \left|R_{m}(\tilde{b} ; x, y)-R_{m}\left(\tilde{b} ; x_{0}, y\right)\right| \\
& \quad \leq C \sum_{|\gamma|<m} \sum_{|\alpha|=m}\left|x-x_{0}\right|^{m-|\gamma|}|x-y|^{|\gamma|}\left\|D^{\alpha} b\right\|_{\operatorname{Lip}_{\beta}(w)} w(\tilde{x}) w\left(2^{k} \tilde{Q}\right)^{\beta / n},
\end{aligned}
$$

thus

$$
\begin{aligned}
& \left|T^{\tilde{b}}\left(f_{2}\right)(x)-T^{\tilde{b}}\left(f_{2}\right)\left(x_{0}\right)\right| \\
& \leq \sum_{k=0}^{\infty} \int_{2^{k+1} \tilde{Q} \backslash 2^{k} \tilde{Q}}\left|R_{m}(\tilde{b} ; x, y)-R_{m}\left(\tilde{b} ; x_{0}, y\right)\right| \frac{|K(x, y)|}{|x-y|^{m}}|f(y)| d y \\
& +\sum_{k=0}^{\infty} \int_{2^{k+1} \tilde{Q} \backslash 2^{k} \tilde{Q}}\left|\frac{K(x, y)}{|x-y|^{m}}-\frac{K\left(x_{0}, y\right)}{\left|x_{0}-y\right|^{m}}\right|\left|R_{m}\left(\tilde{b} ; x_{0}, y\right)\right||f(y)| d y \\
& +C \sum_{|\alpha|=m} \sum_{k=0}^{\infty} \int_{2^{k+1} \tilde{Q} \backslash 2^{k} \tilde{Q}}\left|\frac{K(x, y)}{|x-y|^{m}}-\frac{K\left(x_{0}, y\right)}{\left|x_{0}-y\right|^{m}}\right|\left|(x-y)^{\alpha}\right|\left|D^{\alpha} \tilde{b}(y)\right||f(y)| d y \\
& +C \sum_{|\alpha|=m} \sum_{k=0}^{\infty} \int_{2^{k+1} \tilde{Q} \backslash 2^{k} \tilde{Q}}\left|\frac{(x-y)^{\alpha}}{|x-y|^{m}}-\frac{\left(x_{0}-y\right)^{\alpha}}{\left|x_{0}-y\right|^{m}}\right|\left|K\left(x_{0}, y\right)\right|\left|D^{\alpha} \tilde{b}(y)\right||f(y)| d y \\
& \leq C \sum_{|\alpha|=m}\left\|D^{\alpha} b\right\|_{\operatorname{Lip}_{\beta}(w)} w(\tilde{x}) \sum_{k=0}^{\infty} w\left(2^{k+1} \tilde{Q}\right)^{\beta / n} \\
& \times \int_{2^{k+1} \tilde{Q} \backslash 2^{k} \tilde{Q}}\left(\frac{d}{\left(2^{k} d\right)^{n+1}}+\frac{d^{\varepsilon}}{\left(2^{k} d\right)^{n+\varepsilon}}\right)|f(y)| d y \\
& +C \sum_{|\alpha|=m} \sum_{k=1}^{\infty}\left(\frac{d}{\left(2^{k} d\right)^{n+1}}+\frac{d^{\varepsilon}}{\left(2^{k} d\right)^{n+\varepsilon}}\right) \\
& \times \int_{2^{k} \tilde{Q}}\left|D^{\alpha} b(y)-\left(D^{\alpha} b\right)_{2^{k} \tilde{Q}}\right| w(y)^{-1 / r}|f(y)| w(y)^{1 / r} d y \\
& +C \sum_{|\alpha|=m} \sum_{k=1}^{\infty}\left(\frac{d}{\left(2^{k} d\right)^{n+1}}+\frac{d^{\varepsilon}}{\left(2^{k} d\right)^{n+\varepsilon}}\right) \\
& \times \int_{2^{k} \tilde{Q}}\left|\left(D^{\alpha} b\right)_{2^{k} \tilde{Q}}-\left(D^{\alpha} b\right)_{\tilde{Q}}\right||f(y)| w(y)^{1 / r} w(y)^{-1 / r} d y \\
& \leq C \sum_{|\alpha|=m}\left\|D^{\alpha} b\right\|_{\operatorname{Lip}_{\beta}(w)} w(\tilde{x}) \\
& \times \sum_{k=1}^{\infty}\left(\frac{d}{\left(2^{k} d\right)^{n+1}}+\frac{d^{\varepsilon}}{\left(2^{k} d\right)^{n+\varepsilon}}\right) w\left(2^{k} \tilde{Q}\right)^{\beta / n}\left(\int_{2^{k} \tilde{Q}}|f(y)|^{r} w(y) d x\right)^{1 / r}
\end{aligned}
$$




$$
\begin{aligned}
& \times\left(\frac{1}{\left|2^{k} \tilde{Q}\right|} \int_{2^{k} \tilde{Q}} w(y)^{-1 /(r-1)} d y\right)^{(r-1) / r}\left(\frac{1}{\left|2^{k} \tilde{Q}\right|} \int_{2^{k} \tilde{Q}} w(y) d y\right)^{1 / r}\left|2^{k} \tilde{Q}\right| w\left(2^{k} \tilde{Q}\right)^{-1 / r} \\
& +C \sum_{|\alpha|=m} \sum_{k=1}^{\infty}\left(\frac{d}{\left(2^{k} d\right)^{n+1}}+\frac{d^{\varepsilon}}{\left(2^{k} d\right)^{n+\varepsilon}}\right)\left(\int_{2^{k} \tilde{Q}}\left|\left(D^{\alpha} b(y)-\left(D^{\alpha} b\right)_{2^{k} \tilde{Q}}\right)\right|^{r^{\prime}} w(y)^{1-r^{\prime}} d y\right)^{1 / r^{\prime}} \\
& \times\left(\int_{2^{k} \tilde{Q}}|f(y)|^{r} w(y) d y\right)^{1 / r} \\
& +C \sum_{|\alpha|=m}\left\|D^{\alpha} b\right\|_{\operatorname{Lip}_{\beta}(w)} w(\tilde{x}) \sum_{k=1}^{\infty} k w\left(2^{k} \tilde{Q}\right)^{\beta / n}\left(\frac{d}{\left(2^{k} d\right)^{n+1}}+\frac{d^{\varepsilon}}{\left(2^{k} d\right)^{n+\varepsilon}}\right) \\
& \times\left(\int_{2^{k} \tilde{Q}}|f(y)|^{r} w(y) d x\right)^{1 / r}\left(\frac{1}{\left|2^{k} \tilde{Q}\right|} \int_{2^{k} \tilde{Q}} w(y)^{-1 /(r-1)} d y\right)^{(r-1) / r} \\
& \times\left(\frac{1}{\left|2^{k} \tilde{Q}\right|} \int_{2^{k} \tilde{Q}} w(y) d y\right)^{1 / r}\left|2^{k} \tilde{Q}\right| w\left(2^{k} \tilde{Q}\right)^{-1 / r} \\
& \leq C \sum_{|\alpha|=m}\left\|D^{\alpha} b\right\|_{\operatorname{Lip}_{\beta}(w)} w(\tilde{x}) \sum_{k=1}^{\infty} k\left(2^{-k}+2^{-k \varepsilon}\right)\left(\frac{1}{w\left(2^{k} \tilde{Q}\right)^{1-r \beta / n}} \int_{2^{k} \tilde{Q}}|f(y)|^{r} w(y) d x\right)^{1 / r} \\
& +C \sum_{|\alpha|=m}\left\|D^{\alpha} b\right\|_{\operatorname{Lip}_{\beta}(w)} \\
& \times \sum_{k=1}^{\infty}\left(2^{-k}+2^{-k \varepsilon}\right) \frac{w\left(2^{k} \tilde{Q}\right)}{\left|2^{k} \tilde{Q}\right|}\left(\frac{1}{w\left(2^{k} \tilde{Q}\right)^{1-r \beta / n}} \int_{2^{k} \tilde{Q}}|f(y)|^{r} w(y) d x\right)^{1 / r} \\
& \leq C \sum_{|\alpha|=m}\left\|D^{\alpha} b\right\|_{\operatorname{Lip}_{\beta}(w)} w(\tilde{x}) M_{\beta, r, w}(f)(\tilde{x}) .
\end{aligned}
$$

This completes the proof of Theorem 2 .

Proof of Theorem 3 By putting $1<r<p$ in Theorem 1 and noticing that $w^{1-p} \in A_{1}$, by Lemma 3 and 4, we obtain

$$
\begin{aligned}
\left\|T^{b}(f)\right\|_{L^{p}\left(w^{1-p}\right)} & \leq\left\|M_{\eta}\left(T^{b}(f)\right)\right\|_{L^{p}\left(w^{1-p}\right)} \leq C\left\|M_{\eta}^{\#}\left(T^{b}(f)\right)\right\|_{L^{p}\left(w^{1-p}\right)} \\
& \leq C \sum_{|\alpha|=m}\left\|D^{\alpha} b\right\|_{\mathrm{BMO}(w)}\left\|w M_{r, w}(f)\right\|_{L^{p}\left(w^{1-p}\right)} \\
& =C \sum_{|\alpha|=m}\left\|D^{\alpha} b\right\|_{\mathrm{BMO}(w)}\left\|M_{r, w}(f)\right\|_{L^{p}(w)} \\
& \leq C \sum_{|\alpha|=m}\left\|D^{\alpha} b\right\|_{\mathrm{BMO}(w)}\|f\|_{L^{p}(w)} .
\end{aligned}
$$

This completes the proof of Theorem 3.

Proof of Theorem 4 By choosing $1<r<p$ in Theorem 1 and noticing that $w^{1-p} \in A_{1}$, by Lemmas 5 and 6, we get

$$
\begin{aligned}
\left\|T^{b}(f)\right\|_{L^{p, \varphi}\left(w^{1-p}\right)} & \leq\left\|M_{\eta}\left(T^{b}(f)\right)\right\|_{L^{p, \varphi}\left(w^{1-p}\right)} \leq C\left\|M_{\eta}^{\#}\left(T^{b}(f)\right)\right\|_{L^{p, \varphi}\left(w^{1-p}\right)} \\
& \leq C \sum_{|\alpha|=m}\left\|D^{\alpha} b\right\|_{\mathrm{BMO}(w)}\left\|w M_{r, w}(f)\right\|_{L^{p, \varphi}\left(w^{1-p}\right)}
\end{aligned}
$$




$$
\begin{aligned}
& =C \sum_{|\alpha|=m}\left\|D^{\alpha} b\right\|_{\mathrm{BMO}(w)}\left\|M_{r, w}(f)\right\|_{L^{p, \varphi}(w)} \\
& \leq C \sum_{|\alpha|=m}\left\|D^{\alpha} b\right\|_{\mathrm{BMO}(w)}\|f\|_{L^{p, \varphi}(w)} .
\end{aligned}
$$

This completes the proof of Theorem 4 .

Proof of Theorem 5 By setting $1<r<p$ in Theorem 2 and noting that $w^{1-q} \in A_{1}$, by Lemmas 3 and 4 , we have

$$
\begin{aligned}
\left\|T^{b}(f)\right\|_{L^{q}\left(w^{1-q)}\right.} & \leq\left\|M_{\eta}\left(T^{b}(f)\right)\right\|_{L^{q}\left(w^{1-q}\right)} \leq C\left\|M_{\eta}^{\#}\left(T^{b}(f)\right)\right\|_{L^{q}\left(w^{1-q}\right)} \\
& \leq C \sum_{|\alpha|=m}\left\|D^{\alpha} b\right\|_{\operatorname{Lip}_{\beta}(w)}\left\|w M_{\beta, r, w}(f)\right\|_{L^{q\left(w^{1}-q\right)}} \\
& =C \sum_{|\alpha|=m}\left\|D^{\alpha} b\right\|_{\operatorname{Lip}_{\beta}(w)}\left\|M_{\beta, r, w}(f)\right\|_{L^{q}(w)} \\
& \leq C \sum_{|\alpha|=m}\left\|D^{\alpha} b\right\|_{\operatorname{Lip}_{\beta}(w)}\|f\|_{L^{p}(w)} .
\end{aligned}
$$

This completes the proof of Theorem 5.

Proof of Theorem 6 By choosing $1<r<p$ in Theorem 2, and noticing that $w^{1-q} \in A_{1}$, by Lemmas 5 and 6, we get

$$
\begin{aligned}
\left\|T^{b}(f)\right\|_{L^{q, \varphi}\left(w^{1-q)}\right)} & \leq\left\|M_{\eta}\left(T^{b}(f)\right)\right\|_{L^{q, \varphi}\left(w^{1-q)}\right.} \leq C\left\|M_{\eta}^{\#}\left(T^{b}(f)\right)\right\|_{L^{q, \varphi}\left(w^{1-q}\right)} \\
& \leq C \sum_{|\alpha|=m}\left\|D^{\alpha} b\right\|_{\operatorname{Lip}_{\beta}(w)}\left\|w M_{\beta, r, w}(f)\right\|_{L^{q, \varphi}\left(w^{1-q}\right)} \\
& =C \sum_{|\alpha|=m}\left\|D^{\alpha} b\right\|_{\operatorname{Lip}_{\beta}(w)}\left\|M_{\beta, r, w}(f)\right\|_{L^{q, \varphi}(w)} \\
& \leq C \sum_{|\alpha|=m}\left\|D^{\alpha} b\right\|_{\operatorname{Lip}_{\beta}(w)}\|f\|_{L^{p, \varphi}(w) .}
\end{aligned}
$$

This completes the proof of Theorem 6 .

\section{Competing interests}

The authors declare that they have no competing interests.

\section{Authors' contributions}

The authors completed the paper together. They also read and approved the final manuscript.

\section{Author details}

${ }^{1}$ Department of Mathematics, Huaihua University, Huaihua, Hunan 418008, People's Republic of China. ${ }^{2}$ School of Mathematics and Statistics, Anyang Normal University, Anyang, Henan 455000, People's Republic of China.

Received: 9 January 2014 Accepted: 28 February 2014 Published: 18 Mar 2014

\section{References}

1. Garcia-Cuerva, J, Rubio de Francia, JL: Weighted Norm Inequalities and Related Topics, vol. 16. North-Holland, Amsterdam (1985)

2. Stein, EM: Harmonic Analysis: Real Variable Methods, Orthogonality and Oscillatory Integrals. Princeton University Press, Princeton (1993) 
3. Torchinsky, A: Real Variable Methods in Harmonic Analysis. Pure and Applied Math., vol. 123. Academic Press, New York (1986)

4. Coifman, RR, Rochberg, R, Weiss, G: Factorization theorems for Hardy spaces in several variables. Ann. Math. 103 611-635 (1976)

5. Pérez, C: Endpoint estimate for commutators of singular integral operators. J. Funct. Anal. 128, $163-185$ (1995)

6. Pérez, C, Trujillo-Gonzalez, R: Sharp weighted estimates for multilinear commutators. J. Lond. Math. Soc. 65, 672-692 (2002)

7. Chanillo, S: A note on commutators. Indiana Univ. Math. J. 31, 7-16 (1982)

8. Janson, S: Mean oscillation and commutators of singular integral operators. Ark. Math. 16, $263-270$ (1978)

9. Paluszynski, M: Characterization of the Besov spaces via the commutator operator of Coifman, Rochberg and Weiss Indiana Univ. Math. J. 44, 1-17 (1995)

10. Bloom, S: A commutator theorem and weighted BMO. Trans. Am. Math. Soc. 292, 103-122 (1985)

11. $\mathrm{Hu}, \mathrm{B}, \mathrm{Gu}, \mathrm{J}$ : Necessary and sufficient conditions for boundedness of some commutators with weighted Lipschitz spaces. J. Math. Anal. Appl. 340, 598-605 (2008)

12. He, YX, Wang, YS: Commutators of Marcinkiewicz integrals and weighted BMO. Acta Math. Sinica (Chin. Ser.) 54 513-520 (2011)

13. Zhou, XS, Liu, LZ: Necessary and sufficient conditions for boundedness of commutators of strongly singular integral operators with weighted Lipschitz functions. Indian J. Pure Appl. Math. 42, 405-416 (2011)

14. Cohen, J: A sharp estimate for a multilinear singular integral on $R^{n}$. Indiana Univ. Math. J. 30, 693-702 (1981)

15. Cohen, J, Gosselin, J: On multilinear singular integral operators on $R^{n}$. Stud. Math. 72, 199-223 (1982)

16. Cohen, J, Gosselin, J: A BMO estimate for multilinear singular integral operators. III. J. Math. 30, 445-465 (1986)

17. Coifman, R, Meyer, Y: Wavelets, Calderón-Zygmund and Multilinear Operators. Cambridge Studies in Advanced Math., vol. 48. Cambridge University Press, Cambridge (1997)

18. Ding, Y, Lu, SZ: Weighted boundedness for a class rough multilinear operators. Acta Math. Sin. 17, $517-526$ (2001)

19. Hu, G, Yang, DC: A variant sharp estimate for multilinear singular integral operators. Stud. Math. 141, 25-42 (2000)

20. Garcia-Cuerva, J: Weighted $H^{P}$ spaces. Diss. Math. 162, 1-63 (1979)

21. Peetre, J: On convolution operators leaving $L^{p, \lambda}$-spaces invariant. Ann. Mat. Pura Appl. 72, 295-304 (1966)

22. Peetre, J: On the theory of $L^{p, \lambda}$-spaces. J. Funct. Anal. 4, 71-87 (1969)

23. Di FaZio, G, Ragusa, MA: Commutators and Morrey spaces. Boll. Unione Mat. Ital. 5-A, 323-332 (1991)

24. Di Fazio, G, Ragusa, MA: Interior estimates in Morrey spaces for strong solutions to nondivergence form equations with discontinuous coefficients. J. Funct. Anal. 112, 241-256 (1993)

25. Liu, LZ: Interior estimates in Morrey spaces for solutions of elliptic equations and weighted boundedness for commutators of singular integral operators. Acta Math. Sci. Ser. B 25, $89-94$ (2005)

26. Liu, LZ: Sharp maximal function estimates and boundedness for commutators associated with general integra operator. Filomat 25, 137-151 (2011)

27. Liu, LZ: Multilinear singular integral operators on Triebel-Lizorkin and Lebesgue spaces. Bull. Malays. Math. Sci. Soc. 35, 1075-1086 (2012)

28. Mizuhara, T: Boundedness of some classical operators on generalized Morrey spaces. In: Harmonic Analysis, Proceedings of a Conference Held in Sendai, Japan, pp. 183-189 (1990)

10.1186/1029-242X-2014-115

Cite this article as: Kuang and Wang: Weighted boundedness of multilinear singular integral operators. Journal of Inequalities and Applications 2014, 2014:115

\section{Submit your manuscript to a SpringerOpen ${ }^{\ominus}$ journal and benefit from:}

- Convenient online submission

- Rigorous peer review

- Immediate publication on acceptance

- Open access: articles freely available online

- High visibility within the field

- Retaining the copyright to your article 